PROCEEDINGS OF THE

AMERICAN MATHEMATICAL SOCIETY

Volume 130, Number 7, Pages 2139-2143

S 0002-9939(02)06625-X

Article electronically published on February 27, 2002

\title{
A WEAK ASPLUND SPACE WHOSE DUAL IS NOT IN STEGALL'S CLASS
}

\author{
ONDŘEJ F. K. KALENDA
}

(Communicated by Jonathan M. Borwein)

\begin{abstract}
We show that, under some additional set-theoretical assumptions which are equiconsistent with the existence of a measurable cardinal, there is a weak Asplund space whose dual, equipped with the weak* topology, is not in Stegall's class. This completes a result by Kenderov, Moors and Sciffer.
\end{abstract}

A Banach space $X$ is weak Asplund if every real-valued continuous convex function on $X$ is Gâteaux differentiable at the points of a dense $G_{\delta}$ subset of $X$. This is a large class of Banach spaces which includes many interesting subclasses that are thoroughly studied in [1]. To date, the largest subclass of weak Asplund spaces was introduced by C. Stegall in 15]. This is the class of those Banach spaces $X$ such that $\left(X^{*}, w^{*}\right)$ belongs to the so-called "Stegall class". A topological space $T$ is in Stegall's class if, whenever $B$ is a Baire space and $\varphi$ an upper semicontinuous nonempty compact-valued mapping from $B$ into $T$ which is minimal with respect to inclusion, then $\varphi(b)$ is a singleton for all points $b$ from a dense $G_{\delta}$ subset of $B$.

A further subclass of weak Asplund spaces is that of all Banach spaces $X$ such that $\left(X^{*}, w^{*}\right)$ is fragmentable. Recall that a topological space $(T, \tau)$ is fragmentable if there is a metric $\rho$ on the set $T$ such that any nonempty subset of $T$ has nonempty relatively $\tau$-open subsets of arbitrarily small $\rho$-diameter.

While it is easy to see that any fragmentable topological space is in Stegall's class and any Banach space whose dual, with the weak* topology, is in Stegall's class is weak Asplund, the converse implications were open for a rather long time, see the comments in [1]. In [6] a consistent example of a Stegall compact space which is not fragmentable was constructed. This result was recently improved in [7], where a consistent example was shown of a Banach space $X$ such that $\left(X^{*}, w^{*}\right)$ is in Stegall's class but is not fragmentable.

The main result of the present paper is point (3) of the following theorem. Point (1) is in fact the mentioned result of [7]. Point (2) follows easily from [6] and [7]. We prefer to include these three assertions in one theorem in order to make things more transparent.

Let us further remark that the Theorem below (in fact, already the result of [7]) gives consistent examples of weak Asplund spaces without any Gâteaux smooth

Received by the editors April 5, 2000.

2000 Mathematics Subject Classification. Primary 54C60, 26E25, 54C10.

Key words and phrases. Weak Asplund space, fragmentable space, Stegall's class of spaces.

Partially supported by research grants GAUK 277/2001, GAČR 201/00/1466 and MSM 113200007. 
Lipschitz bump function. Indeed, if $X$ has a Gâteaux smooth norm 12 or at least a Gâteaux smooth Lipschitz bump function [2], [9], then $X^{*}$ is weak* fragmentable.

Theorem. (1) If $\aleph_{1}=\aleph_{1}^{L}$, then there is a Banach space $X$ such that $\left(X^{*}, w^{*}\right)$ is in Stegall's class but not fragmentable.

(2) If Martin's axiom and the negation of the continuum hypothesis hold, then there is a weak Asplund space $X$ such that $\left(X^{*}, w^{*}\right)$ is not fragmentable.

(3) If there is a precipitous ideal on $\omega_{1}$ and Martin's axiom and the negation of the continuum hypothesis hold, then there is a weak Asplund space $X$ such that $\left(X^{*}, w^{*}\right)$ is not in Stegall's class.

Let us make a few remarks on the axioms used. The letter $L$ denotes the constructible universe. So $\aleph_{1}=\aleph_{1}^{L}$ is satisfied for example under the axiom of constructibility (i.e., $V=L$ ) but also in other situations (cf. 6]). For the definition of a precipitous ideal we refer to [4] or [3]. Its existence on $\omega_{1}$ is equiconsistent with the existence of a measurable cardinal. Further, it follows from [5] and [14 that if it is consistent to suppose that there is a measurable cardinal, then the assumptions of (3) are consistent. Indeed, if it is consistent that there is a measurable cardinal, then, due to [4], it is consistent to suppose there is a normal precipitous ideal on $\omega_{1}$. Now apply the Theorem in section 7.11 of 14 to get a complete Boolean algebra satisfying the countable chain condition which yields an extension in which Martin's axiom and the negation of the continuum hypothesis hold. Finally, such an extension preserves normal precipitous ideals on $\omega_{1}$ due to [5]. Notice also that the assumptions of (1) and (3) cannot hold simultaneously.

To prove the main result we need to introduce, consistently with [6], the following subclasses of Stegall's class. Let $\mathcal{C}$ be a class of Baire spaces which is closed with respect to taking open subsets and dense Baire subspaces. We say that a topological space $T$ is in Stegall's class with respect to the class $\mathcal{C}$ if, whenever $B$ is from $\mathcal{C}$ and $\varphi$ is an upper semicontinuous nonempty compact-valued mapping from $B$ into $T$ which is minimal with respect to inclusion, then $\varphi(b)$ is a singleton for all points $b$ from a dense $G_{\delta}$ subset of $B$.

Let us remark that $T$ is in Stegall's class if and only if it is in Stegall's class with respect to the class of all Baire metric spaces by [8]. Further, if $X$ is a Banach space such that $\left(X^{*}, w^{*}\right)$ is in Stegall's class with respect to the class of all Baire metric spaces with density at most equal to the density of $X$, then $X$ is weak Asplund; cf. [1, Theorem 3.2.2 and its proof].

Further we recall the construction used in [6] and then in [7]. Let $A \subset(0,1)$ be an arbitrary subset. Put

$$
K_{A}:=((0,1] \times\{0\}) \cup((\{0\} \cup A) \times\{1\})
$$

and equip this set with the lexicographical order (i.e., $(s, a)<(t, b)$ if either $s<t$, or $s=t$ and $a<b$ ) and the order topology. Then $K_{A}$ is a compact Hausdorff space, see [6, Proposition 2]. Now we give the key proposition.

Proposition. Let $\mathcal{C}$ be a class of Baire metric spaces which is closed with respect to taking open subspaces and dense Baire subspaces, and suppose that $A \subset(0,1)$. Then the following assertions are equivalent.

(a) $\left(C\left(K_{A}\right)^{*}, w^{*}\right)$ is in Stegall's class with respect to $\mathcal{C}$.

(b) $K_{A}$ is in Stegall's class with respect to $\mathcal{C}$.

(c) For any $B \in \mathcal{C}$ and any continuous function $f: B \rightarrow A$ the function $f$ has at least one local extremum, i.e., a local minimum or a local maximum. 
(d) For any $B \in \mathcal{C}$ and any continuous function $f: B \rightarrow A$ there is a nonempty open $U \subset B$ such that $f$ is constant on $U$.

Proof. The implication (a) $\Rightarrow$ (b) is trivial, as $K_{A}$ is homeomorphic to a subspace of $\left(C\left(K_{A}\right)^{*}, w^{*}\right)$.

The equivalence of (b) and (c) is proved in [6, Proposition 4].

The implication $(\mathrm{d}) \Rightarrow(\mathrm{a})$ is proved in [7].

So it remains to show that $(\mathrm{c}) \Rightarrow(\mathrm{d})$. Suppose that (c) holds and that there is some $B \in \mathcal{C}$ and a continuous function $f: B \rightarrow A$ which is not constant on any nonempty open subset of $B$. Fix a metric $\rho$ generating the topology of $B$. For $n \in \mathbb{N}$ put

$$
\begin{gathered}
M_{n}^{\geq}:=\left\{b \in B: f(b)=\max \left\{f\left(b^{\prime}\right): \rho\left(b, b^{\prime}\right)<1 / n\right\}\right\}, \\
M_{n}^{\leq}:=\left\{b \in B: f(b)=\min \left\{f\left(b^{\prime}\right): \rho\left(b, b^{\prime}\right)<1 / n\right\}\right\} .
\end{gathered}
$$

Then clearly both of the sets $M_{n}^{\geq}$and $M_{n}^{\leq}$are closed. If one of these sets has an interior point, then $f$ is constant on a neighborhood of it. Indeed, if $b$ is an interior point of $M_{n}^{\geq}$, then $B_{\rho}(b, \delta) \subset M_{n}^{\geq}$for some $\delta>0$. Let $b^{\prime} \in B$ be such that $\rho\left(b, b^{\prime}\right)<\min \left(\delta, \frac{1}{n}\right)$. Then both $f(b) \geq f\left(b^{\prime}\right)$ and $f\left(b^{\prime}\right) \geq f(b)$, so $f\left(b^{\prime}\right)=f(b)$.

Hence both of the sets $M_{n}^{\geq}$and $M_{n}^{\leq}$are closed and nowhere dense. Therefore, $M:=\bigcup_{n \in \mathbb{N}}\left(M_{n}^{\geq} \cup M_{n}^{\leq}\right)$is a meager set and hence has an empty interior. Thus $B^{\prime}:=B \backslash M$ is a dense Baire subspace of $B$; therefore it belongs to $\mathcal{C}$. So, by (c), $f \uparrow B^{\prime}$ has a local extremum at a point $b$. Then, by the continuity of $f$ and the density of $B^{\prime}$ in $B, f$ has a local extremum at $b$, with respect to $B$, too. Thus $b \in M$, a contradiction.

Proof of the Theorem. (1) Suppose $\aleph_{1}=\aleph_{1}^{L}$. By [13] there is an uncountable coanalytic set $A \subset(0,1)$ which has no perfect subset. By [11, Lemma 5.1] this $A$ satisfies condition (d) of the Proposition if $\mathcal{C}$ is the class of all Hausdorff completely regular Baire spaces. By the Proposition $\left(C\left(K_{A}\right)^{*}, w^{*}\right)$ is in Stegall's class with respect to the class of all Baire metric spaces, and so it is in Stegall's class by 8 . Further, $\left(C\left(K_{A}\right)^{*}, w^{*}\right)$ is not fragmentable, as $K_{A}$ is not fragmentable by [6] Proposition 3].

(2) Suppose Martin's axiom and the negation of the continuum hypothesis hold. Then there is (by [10, p.162]) a set $A \subset(0,1)$ of cardinality $\aleph_{1}$ such that each subset of $A$ is relatively $F_{\sigma}$. By [6] Proposition 7(1)] the set $A$ satisfies condition (d) of the Proposition if $\mathcal{C}$ is the class of all Baire spaces of weight at most $\aleph_{1}$. Hence, by the Proposition, $\left(C\left(K_{A}\right)^{*}, w^{*}\right)$ is in Stegall's class with respect to the class of all metrizable Baire spaces of weight at most $\aleph_{1}$. Moreover, $C\left(K_{A}\right)$ has density $\aleph_{1}$, so it is weak Asplund. Again, $\left(C\left(K_{A}\right)^{*}, w^{*}\right)$ is not fragmentable, as $K_{A}$ is not fragmentable by [6, Proposition 3].

(3) Suppose there is a precipitous ideal on $\omega_{1}$ and Martin's axiom and the negation of the continuum hypothesis hold. By point (2) there is an uncountable $A \subset(0,1)$ such that $C\left(K_{A}\right)$ is weak Asplund. However, by [11] (or [6, Proposition 8]) there are no uncountable subsets of $(0,1)$ that satisfy condition $(d)$ of the Proposition with respect to metrizable Baire spaces of weight at most $2^{\aleph_{1}}$. Hence, by the Proposition, $\left(C\left(K_{A}\right)^{*}, w^{*}\right)$ is not in Stegall's class.

Now we collect some open problems. First, notice that the axioms used in assertions (1) and (3) of the Theorem cannot hold simultaneously. Indeed, if there is a precipitous ideal on $\omega_{1}$, then it follows from Theorem 3.2 and the Lemma in 
the proof of Theorem 3.3 in [3], together with [16], that $\aleph_{1}>\aleph_{1}^{L}$. So the following question is natural.

Question 1. Is it consistent that all three classes - weak Asplund spaces, Banach spaces whose dual, with the weak* topology, lie in Stegall's class, and Banach spaces with weak* fragmentable dual - are mutually distinct?

The following question has a positive answer within the spaces $K_{A}$.

Question 2. Suppose $K$ is a Stegall compact space. Is $\left(C(K)^{*}, w^{*}\right)$ in Stegall's class as well?

The next question concerns a possible strengthening of assertion (3) of the Theorem.

Question 3. Is there a weak Asplund space $X$ with density $\aleph_{1}$ such that $\left(X^{*}, w^{*}\right)$ is not in Stegall's class with respect to the class of metrizable Baire spaces of weight at most $\aleph_{1}$ ?

Finally, we can ask about the necessity of some of the set-theoretical assumptions.

Question 4. Is it consistent that fragmentable and Stegall compact spaces coincide? Is it true if there is a precipitous ideal on $\omega_{1}$ ? Is it consistent that each weak Asplund space has a weak* fragmentable dual? Is it true if there is a precipitous ideal on $\omega_{1}$ and the continuum hypothesis holds?

The author is grateful to B. Balcar for helpful consultations on forcing.

\section{ADDED IN PROOF}

A positive answer to Question 1 was obtained by K. Kunen and the author.

\section{REFERENCES}

[1] M.Fabian, Gâteaux differentiability of convex functions and topology: weak Asplund spaces, Wiley-Interscience, New York, 1997. MR 98h:46009

[2] M.Fosgeram, PhD thesis, Univ. College London, 1992, pp. 52-62.

[3] R.Frankiewicz and K.Kunen, Solution of Kuratowski's problem on function having the Baire property I., Fund. Math. 128 (1987), 171-180. MR 89a:03090

[4] T.Jech, M.Magidor, W.Mitchell and K.Prikry, Precipitous ideals, J. Symb. Log. 45 (1980), 1-8. MR 81h:03097

[5] Y.Kakuda, On a condition for Cohen extensions which preserve precipitous ideals, J. Symb. Log. 46 (2) (1981), 296-300. MR 82i:03062

[6] O.Kalenda, Stegall compact spaces which are not fragmentable, Topol. Appl. 96 (2) (1999), 121-132. MR 2001i: 45027

[7] P.Kenderov, W.Moors and S.Sciffer, A weak Asplund space whose dual is not weak* fragmentable, Proc. Amer. Math. Soc. 129 (2001), 3741-3747.

[8] P.Kenderov and J.Orihuela, A generic factorization theorem, Mathematika 42 (1) (1995), 56-66. MR 96h:54014

[9] I.Kortezov, Fragmentability of the dual of a Banach space with smooth bump, Serdica Math. J. 24 (2) (1998), 187-198. MR 2000b:46028

[10] D.A.Martin and R.M.Solovay, Internal Cohen extensions, Ann. Math. Logic 2 (1970), 143-178. MR 42:5787

[11] I.Namioka and R.Pol, Mappings of Baire spaces into function spaces and Kadec renorming, Israel Jour. Math. 78 (1992), 1-20. MR 94f:46020

[12] N.K.Ribarska, The dual of a Gâteaux smooth Banach space is weak star fragmentable, Proc. Amer. Math. Soc. 114 (4) (1992), 1003-1008. MR 92g:46020 
[13] R.M.Solovay, On the cardinality of $\Sigma_{2}^{1}$ sets of reals, Foundation of Mathematics, Symposium papers commemorating the sixtieth birthday of Kurt Gödel, Springer-Verlag, 1969, pp. 58-73. MR 43:3115

[14] R.M.Solovay and S.Tennenbaum, Iterated Cohen extensions and Souslin's problem, Ann. of Math. 94 (1971), 201-245. MR 45:3212

[15] C. Stegall, A class of topological spaces and differentiability, Vorlesungen aus dem Fachbereich Mathematik der Universität Essen 10 (1983), 63-77. MR 85j:46026

[16] S.Ulam, Zur Masstheorie in der allgemeinen Mengenlehre, Fund. Math. 16 (1930), 140-150.

Department of Mathematical Analysis, Sokolovská 83, 18675 Praha 8, Czech RepubLIC

E-mail address: kalenda@karlin.mff.cuni.cz 